Supporting Information For

\title{
Lithium-Ion Battery Cycling for Magnetism Control
}

\author{
Qingyun Zhang, ${ }^{1, \dagger}$ Xi Luo, ${ }^{1 \dagger}$ Luning Wang, ${ }^{2}$ Lifang Zhang, ${ }^{2}$ Bilal Khalid, ${ }^{1}$ \\ Jianghong Gong, ${ }^{1}$ and Hui $\mathrm{Wu}^{1, *}$
}

${ }^{1}$ State Key Laboratory of New Ceramics and Fine Processing, School of Materials

Science and Engineering, Tsinghua University, Beijing, 100084, China

${ }^{2}$ School of Materials Science and Engineering, University of Science and Technology

Beijing, Beijing, 100083, China

Email: huiwu@tsinghua.edu.cn

This file includes:

Materials and Methods

Supplementary Text

Figs. S1 to S6

Table S1

Video S1

References 


\section{Materials and methods}

\section{Battery design and fabrication}

The experimental device was designed by a pouch lithium ion battery. As shown in Figure $\mathrm{S} 1$, the pouch cell consists of $\mathrm{Cu}$-foil, metallic $\mathrm{Li}$-foil, separator as well as PET sheets. The positive electrode active materials contain $80 \mathrm{wt} \%$ nanoscale ferric oxide particle $\left(\alpha-\mathrm{Fe}_{2} \mathrm{O}_{3}, 50 \mathrm{~nm}\right), 10 \mathrm{wt} \%$ conducting carbon (Super P) and $10 \mathrm{wt} \%$ sodium carboxymethyl-cellulose (CMC, 9000), which were coated on the Cu-foil. The metallic $\mathrm{Li}$ was used as counter and reference electrode. The lithium hexafluorophosphate (LiPF6) dispersed in propylene carbonate (PC) was used as electrolyte. The Polyethylene terephthalate (PET) sheets were utilized to seal the battery compositions and make the battery flexible for subsequent VSM measurements. Such materials as well as separator and electrolyte were assembled to be a pouch cell in an argon-filled glove box. Here, it must be mentioned that each pre-designed battery used as experimental device includes $\mathrm{Cu}$ foil loaded nanoscale $\alpha-\mathrm{Fe}_{2} \mathrm{O}_{3}$ mass of about $0.82 \mathrm{mg}$ within the area of $16 \mathrm{~mm}^{2}$, and the whole device was confined with the length and width being 35 and $25 \mathrm{~mm}$, respectively.

\section{Measurements of electrochemical performance}

Figure S2a shows discharge voltage profiles for the first to tenth galvanostatic cycles of experimental device between 3.0 and $0 \mathrm{~V}$ at testing current of $200 \mathrm{~mA} / \mathrm{g}$ performed on the electrochemical workstation (BTS-610). The total initial discharge capacity is around $1246 \mathrm{mAh} / \mathrm{g}$, presenting higher than the theoretical capacity (1007 
$\mathrm{mAh} / \mathrm{g})^{1}$ of $\mathrm{Fe}_{2} \mathrm{O}_{3}$, which is ascribed to its structure with nanoscale ${ }^{2}$. And, the specific capacity could remain to be approximately $832 \mathrm{mAh} / \mathrm{g}$ after 10 cycles. Combining the Figure S2b, after cycling for 20 discharge-charge processes, the discharge and charge capacity could still keep value of 706 and $640 \mathrm{mAh} / \mathrm{g}$, respectively. Such above electrochemical parameters confirmed that the pre-designed pouch battery can function well during the discharge-charge cycling process. Additionally, it is important to mention that the pouch cell presents a typical flat discharge plateau at approximately $0.7 \mathrm{~V}$ observed from the first discharge curve in Figure S2a, which would contribute to the operation of tuning voltage to control magnetization in the next experimental section. Such above electrochemical parameters confirmed that the pre-designed pouch battery can function well during the discharge-charge cycling process.

\section{VSM measurements on three pristine materials}

To clearly demonstrate the significant changes of magnetization during the lithiation-delithiation process, we performed the vibration sample magnetometer (VSM) on the magnetic measurement of row material $\alpha-\mathrm{Fe}_{2} \mathrm{O}_{3}$ nanoparticle and as-prepared electrode paste coated on the $\mathrm{Cu}$ foil under an applied magnetic field of $10 \mathrm{kOe}$ at room temperature. As shown in Figure S3, the hysteresis loops of above two pristine samples were similar to that of pre-designed device at the starting first discharge voltage of $3.0 \mathrm{~V}$, especially in the saturation magnetization $M_{\mathrm{S}}$. Meanwhile, the hysteresis loops of pristine pre-designed device was not so smooth, which was 
attributed to the small quantity of electrode active material included in the device and the fairly weak magnetization of $\alpha-\mathrm{Fe}_{2} \mathrm{O}_{3}$ (theoretical $M_{\mathrm{S}}$ value of $0.4307 \mathrm{emu} / \mathrm{g}$ for $80 \mathrm{~nm}$ particles ${ }^{3}$ ). In addition, only few changes in the coercive force $H_{\mathrm{C}}$ of the three pristine samples can be observed in the insert image of the Figure S3. Moreover, the specific magnetic parameters of three pristine samples were further given in Table S1. The pre-designed device at first-discharge voltage of $3.0 \mathrm{~V}$ presents quite weak in the $M_{\mathrm{S}}(0.900 \mathrm{emu} / \mathrm{g})$, is in agreement with that of pristine $\alpha-\mathrm{Fe}_{2} \mathrm{O}_{3}$ nanoparticles $(0.862$ $\mathrm{emu} / \mathrm{g})$.

\section{SEM and EDS measurements on pristine materials}

It is assumed that the micro-structure and shape size of the $\alpha-\mathrm{Fe}_{2} \mathrm{O}_{3}$ particles have been found to be an important factor in determining the magnetic properties and electrochemical performances ${ }^{4}$. Figure $\mathrm{S} 4 \mathrm{a}$ is an example of a low magnification SEM image showing the morphology and size distribution of raw $\alpha-\mathrm{Fe}_{2} \mathrm{O}_{3}$ particles. And, the higher magnification SEM (Fig. S4b) further confirm the pristine $\alpha-\mathrm{Fe}_{2} \mathrm{O}_{3}$ represents a uniform spherical particle with a diameter of approximately $50 \mathrm{~nm}$, which would make it possible to intercalate more $\mathrm{Li}$ into $\mathrm{Fe}_{2} \mathrm{O}_{3}$ during the lithiation process and enhance lager variation on the magnetization of the experimental device. In addition, as shown in Figure $\mathrm{S} 4 \mathrm{c}$, the crystalline phase of pristine $\mathrm{Fe}_{2} \mathrm{O}_{3}$ particles was further identified as the alpha- $\mathrm{Fe}_{2} \mathrm{O}_{3}$ (PDF card No.89-598), and the chemical composition of the alpha- $\mathrm{Fe}_{2} \mathrm{O}_{3}$ particles was also checked by EDS (Fig. S4d), which demonstrates that it consists of $\mathrm{Fe}$ and $\mathrm{O}$. 


\section{CV measurements}

Cyclic voltammograms were recorded on the pouch battery with $\mathrm{Fe}_{2} \mathrm{O}_{3}$-based electrode in the voltage from 3.0 to $0.0 \mathrm{~V}$ at a scan rate of $0.5 \mathrm{mV} \mathrm{s}^{-1}$. As shown in Figure S5, a visibly broad cathodic peak at potential of $0.71 \mathrm{~V}$ in $1^{\text {st }}$ cycle is indication of the single-phase insertion of $\mathrm{Li}$ and reduction of $\mathrm{Fe}^{3+}$ in $\alpha-\mathrm{Fe}_{2} \mathrm{O}_{3}$ nanoparticles ${ }^{5}$, which agrees well with the first discharging voltage plateau at $0.70 \mathrm{~V}$ (Fig. S2a) and result that the phase of $\mathrm{LiFeO}_{2}$ presents at about $0.70 \mathrm{~V}$ (see Fig. 3). The subsequent anodic peak at about $1.80 \mathrm{~V}$ corresponds to the voltage plateau in the first galvanostatic charging curve (see Fig. 2a). The second cycle varies from the first one, a sharp cathodic peak is indicated by a smooth sloping curve from 1.23 to $0.85 \mathrm{~V}$, which is in agreement with the second discharging voltage plateau (Fig. S2a). And the position of second anodic peak shifts towards about $1.72 \mathrm{~V}$. It is commonly accepted that such changes in the CVs are caused by the conversion reaction, which occurs during the lithiation-delithiation process ${ }^{6}$. 


\section{Supplementary Text}

1. Supplementary VSM measurements on the pouch cell during the fifth discharge-charge cycle

In the main manuscript, Fig. 4 demonstrates that a switching effect could be achieved through Li-ion battery (LIB) cycling between 2.0 and $0.5 \mathrm{~V}$. Here, to investigate whether the magnetization-demagnetization process is reversible and is similar to the first discharge-charge cycle, we subjected the experimental device to several complete discharge-charge cycles. We then selected six potential (Fig. S6a) during a different fifth discharge and charge state to investigate magnetization changes of the device using VSM measurements at room temperature. Figures S6b and S6c show the changes in magnetization of the experimental device. The hysteresis loops with a significant improvement (Fig. S6b) and a reduction (Fig. S6c) in $M_{\mathrm{S}}$ were collected during the fifth lithiation-delithiation process. These results are consistent with the phenomenon observed in the first lithiation/delithiation reaction (Fig. $2 b$ and 2c).

Figure S6d further records the $M_{\mathrm{S}}$ and $H_{\mathrm{C}}$ changes in detail. Here, we noted that $M_{\mathrm{S}}(3.46 \mathrm{emu} / \mathrm{g})$ recorded at $2.3 \mathrm{~V}$ in the fifth lithiation was larger by 1 order of magnitude than the $M_{\mathrm{S}}(0.9 \mathrm{emu} / \mathrm{g})$ recorded at $3.0 \mathrm{~V}$ in the fifth lithiation process. This result is attributed to the complete conversion mechanism from $\mathrm{Fe}_{2} \mathrm{O}_{3}$ to $\mathrm{Fe}$ after the initial lithiation and delithiation processes. This mechanism is described as the following reaction ${ }^{6}$ : 


$$
\begin{aligned}
\mathrm{Fe}_{2} \mathrm{O}_{3}+6 \mathrm{Li}^{+} & +6 e^{-} \stackrel{1 \text { st lithiation }}{\longrightarrow} 3 \mathrm{Li}_{2} \mathrm{O}+2 \mathrm{Fe} \\
& \stackrel{\text { delithiation }\left(-4.5 \mathrm{Li}^{+}\right) / \text {/ithiation }\left(+4.5 \mathrm{Li}^{+}\right)}{\Longleftrightarrow} 1.5 \mathrm{LiFeO}_{2}+0.5 \mathrm{Fe}
\end{aligned}
$$

Starting from the second cycle, the lithiation and delithiation reaction occurs because of the $\mathrm{LiFeO}_{2}$ to $\mathrm{Fe}$ conversion. Therefore, at the beginning of the fifth discharge process, most of the $\mathrm{LiFeO}_{2}$ in the experimental device is converted to Fe instead of reverting to $\mathrm{Fe}_{2} \mathrm{O}_{3}$. This process explains why the $M_{\mathrm{S}}$ (collected at $2.6 \mathrm{~V}$ ) during the fifth lithiation process is higher than the $M_{\mathrm{S}}$ obtained at $3.0 \mathrm{~V}$ during the first lithiation process. In addition, $M_{\mathrm{S}}$ increases while $H_{\mathrm{C}}$ decreases during fifth lithiation process, and $M_{\mathrm{S}}$ decreases while $H_{\mathrm{C}}$ increases during fifth delithiation process. This response is similar to observations (Fig. 2d) in the first lithiation and delithiation processes. Consequently, realization of reversible magnetization and demagnetization through lithiation/delithiation reaction after five discharge-charge cycles confirms that the process can be controlled during each discharge-charge cycles. This result meets the expectations for multi-reversible magnetization manipulation based on the voltage-tuning lithiation-delithiation state.

\section{The amount change of $\mathrm{Fe}_{2} \mathrm{O}_{3}$ related to XRD spectrum during first discharge process}

In the main manuscript, we provided the phase change of electrode (Figure 3) to investigate the mechanism of magnetized phenomenon during first discharge process. To further confirm the relevance between phase change of electrode and magnetized phenomenon, we calculate the amount change of $\mathrm{Fe}_{2} \mathrm{O}_{3}$ via the capacity of LIB. The 
capacity of the LIB is highly related to the amount change of reactive electrode materials, which can be shown by following formula:

$$
C_{0}=26.8 n \frac{m_{0}}{M}
$$

Where $C_{0}$ is the capacity and $m_{0}$ the mass of reacted electrode, the $n$ is number of reactive electronics. Basing on the capacity change obtained from Figure 2a during the discharging process, the amount of $\mathrm{Fe}_{2} \mathrm{O}_{3}$ has been in reaction can be acquired by formula (1). Therefore, it is reasonable to gain the remainder amount change of $\mathrm{Fe}_{2} \mathrm{O}_{3}$ (Table S2). During the first discharging process, the remainder $\mathrm{Fe}_{2} \mathrm{O}_{3}$ gradually decreased, which corresponds to the XRD patterns change (Figure 3).

\section{Video of the observed experimental phenomenon}

To further illustrate the voltage-tuning magnetization phenomenon, we recorded a short video demonstrating the magnetization change during the first discharge-charge process. In this video, the pouch battery is being discharged/charged and the magnet is fixed opposite the pouch cell. At the beginning of the discharge process, no visible phenomenon is observed because the magnetism is weak. Nevertheless, after the discharge process, the pouch battery starts to gradually swing back and forth. At the end of the discharge process, the pouch cell is attached to the magnet. 


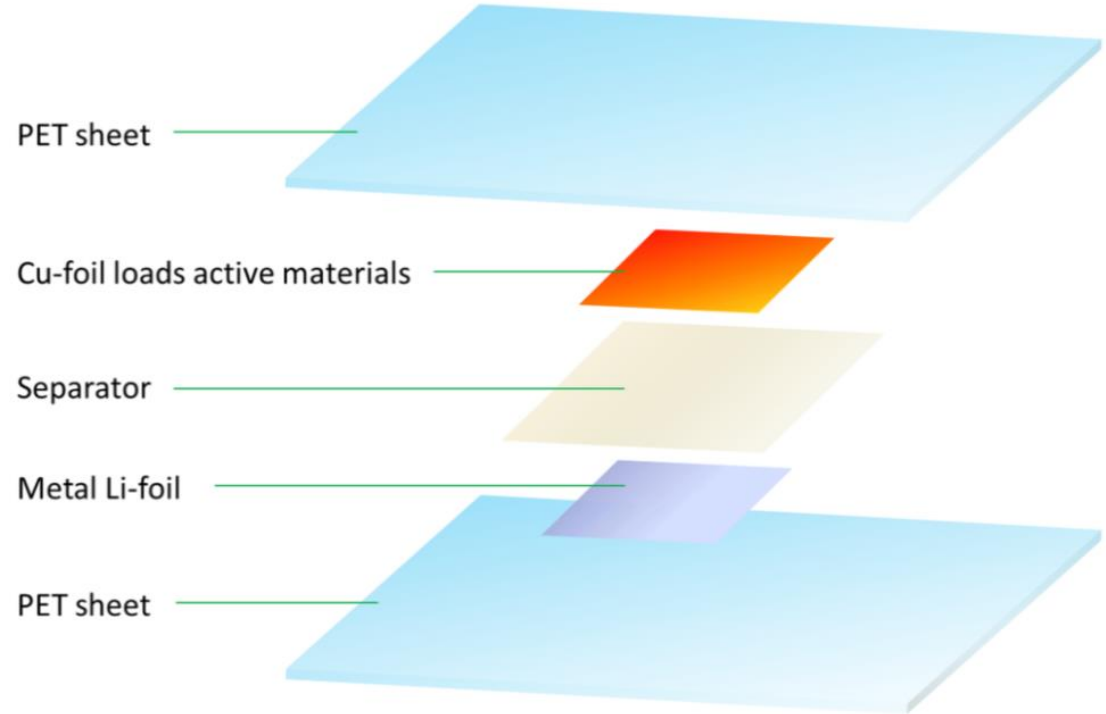

Figure S1. Exploded-view illustration of the pouch battery device. 

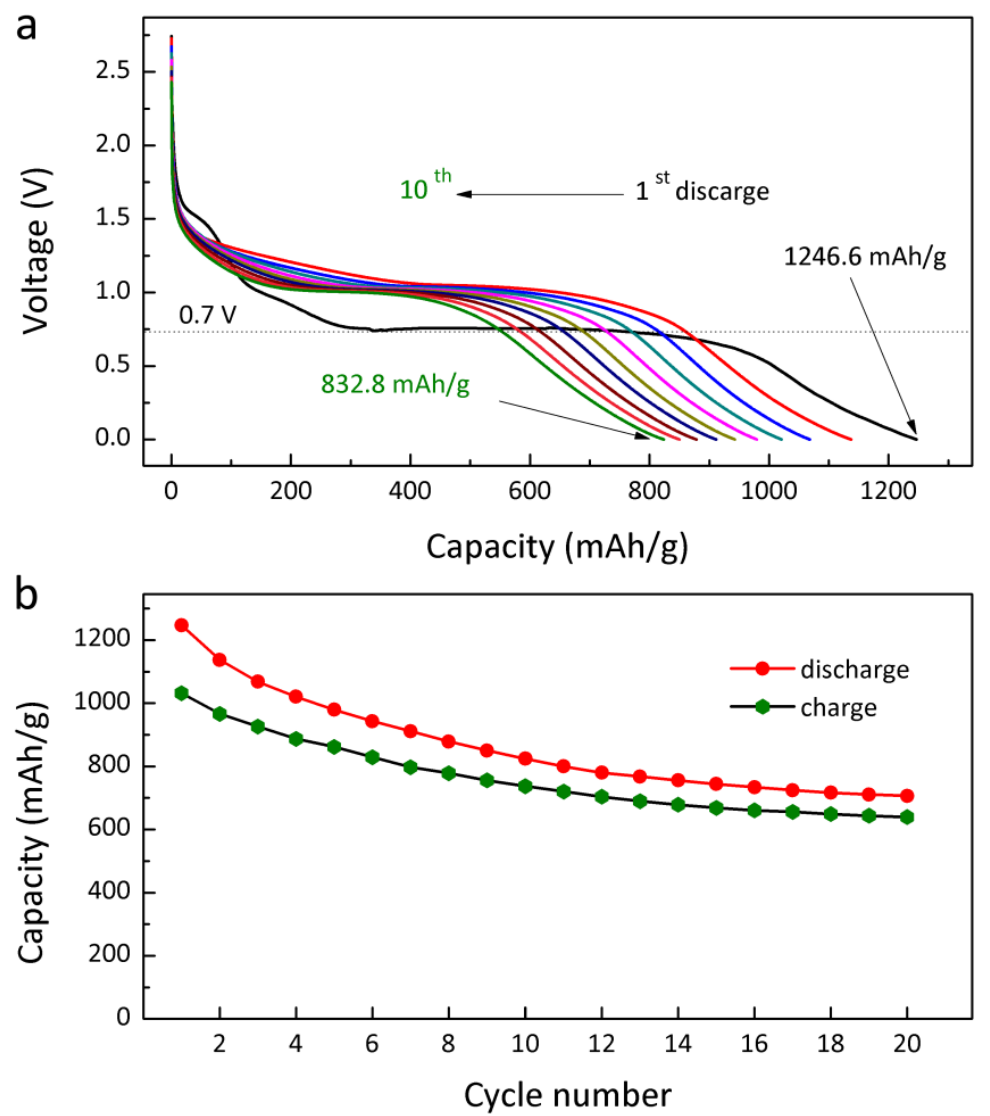

Figure S2. (a) First to tenth discharge curves of the pouch cell based on $\mathrm{Li} / \alpha-\mathrm{Fe}_{2} \mathrm{O}_{3}$ cycled between 3.0 and $0 \mathrm{~V}$ vs.Li/ $/ \mathrm{Li}^{+}$at the current density of $200 \mathrm{~mA} / \mathrm{g}$. (b) Cycling performance of the pouch battery at testing current of $200 \mathrm{~mA} / \mathrm{g}$ for 20 cycles. 


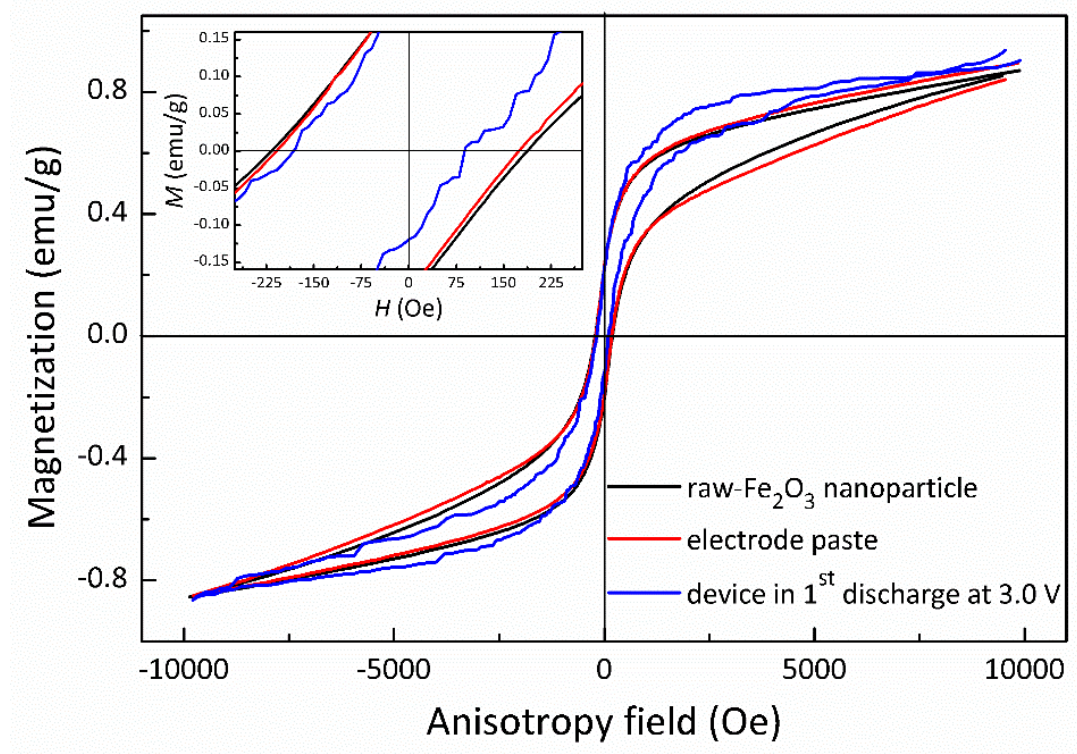

Figure S3. Magnetic hysteresis loops of the $\alpha-\mathrm{Fe}_{2} \mathrm{O}_{3}$ raw nanoparticles, as-prepared electrode paste, and pristine $\alpha-\mathrm{Fe}_{2} \mathrm{O}_{3}$-based electrode at the first discharge voltage of $3.0 \mathrm{~V}$ and at room temperature. 

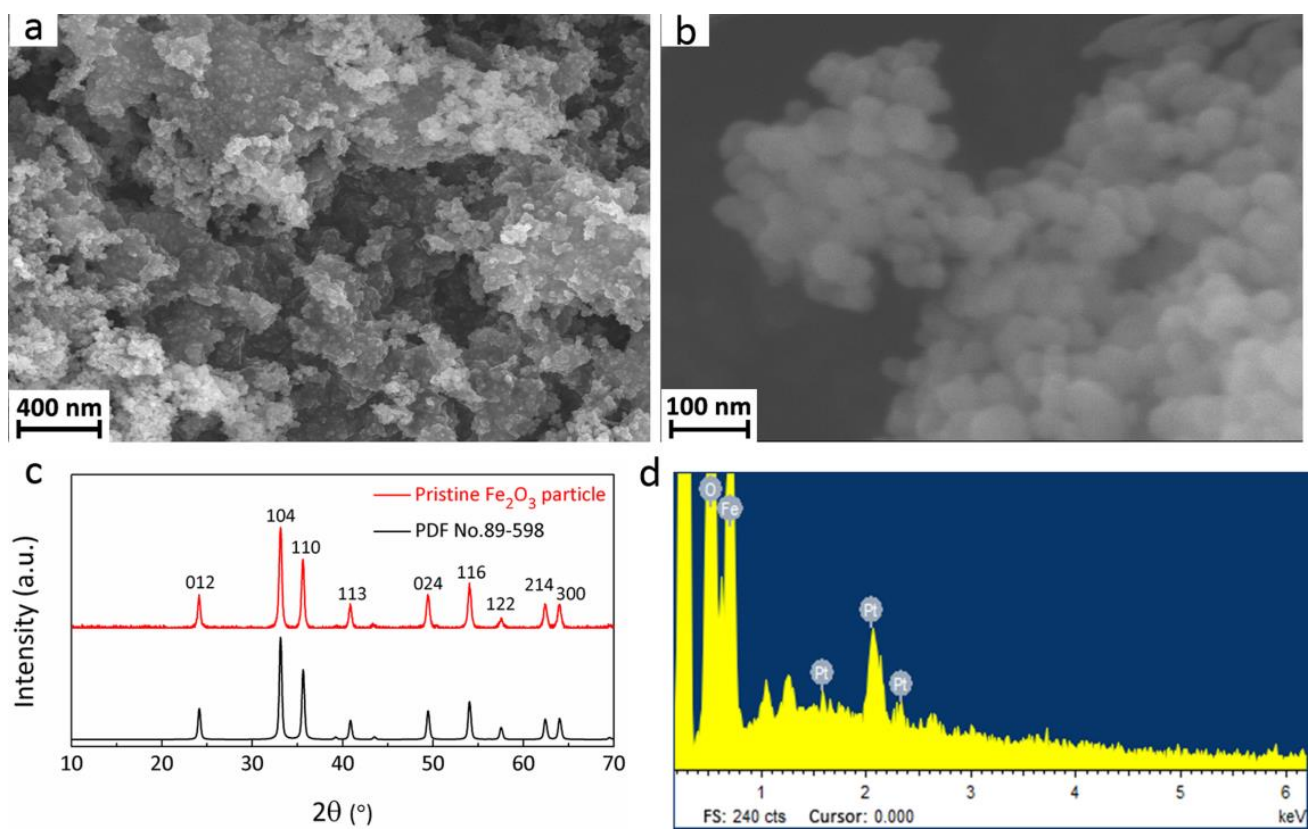

Figure S4. (a,b) Low and higher magnification SEM of the row $\alpha-\mathrm{Fe}_{2} \mathrm{O}_{3}$ nanoparticles. (c,d) XRD pattern and EDS spectrum of the pristine $\alpha-\mathrm{Fe}_{2} \mathrm{O}_{3}$ particles. 


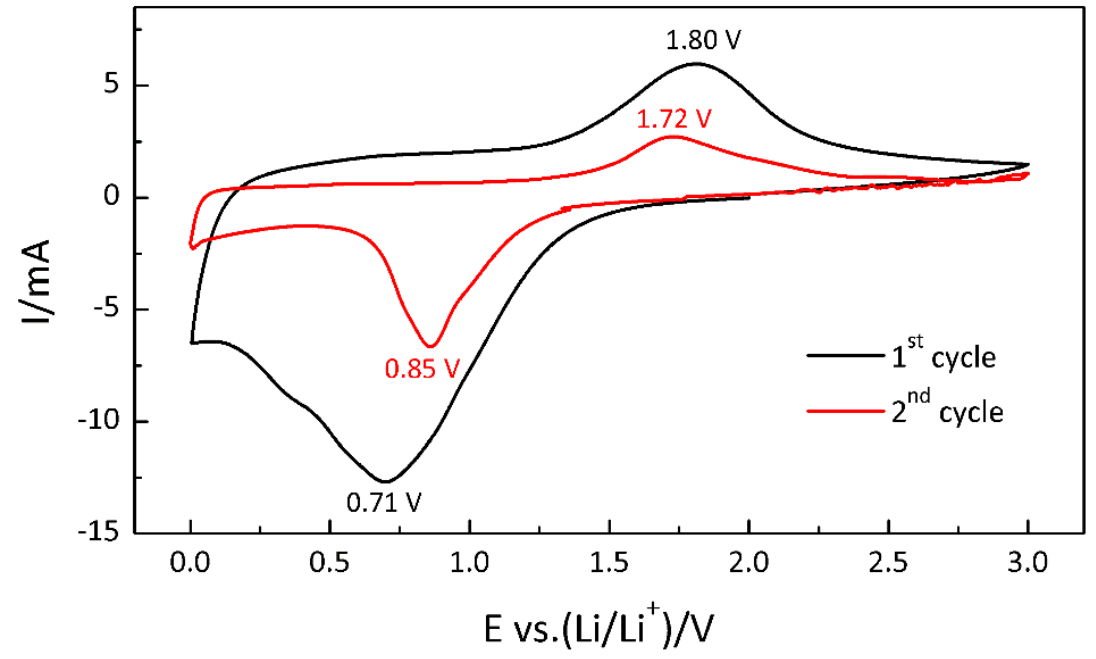

Figure S5. Cyclic voltammograms of the $\alpha-\mathrm{Fe}_{2} \mathrm{O}_{3}$ nanoparticle-based electrode cycled between 3.0 and $0.0 \mathrm{~V}$ vs. $\mathrm{Li} / \mathrm{Li}^{+}$. 

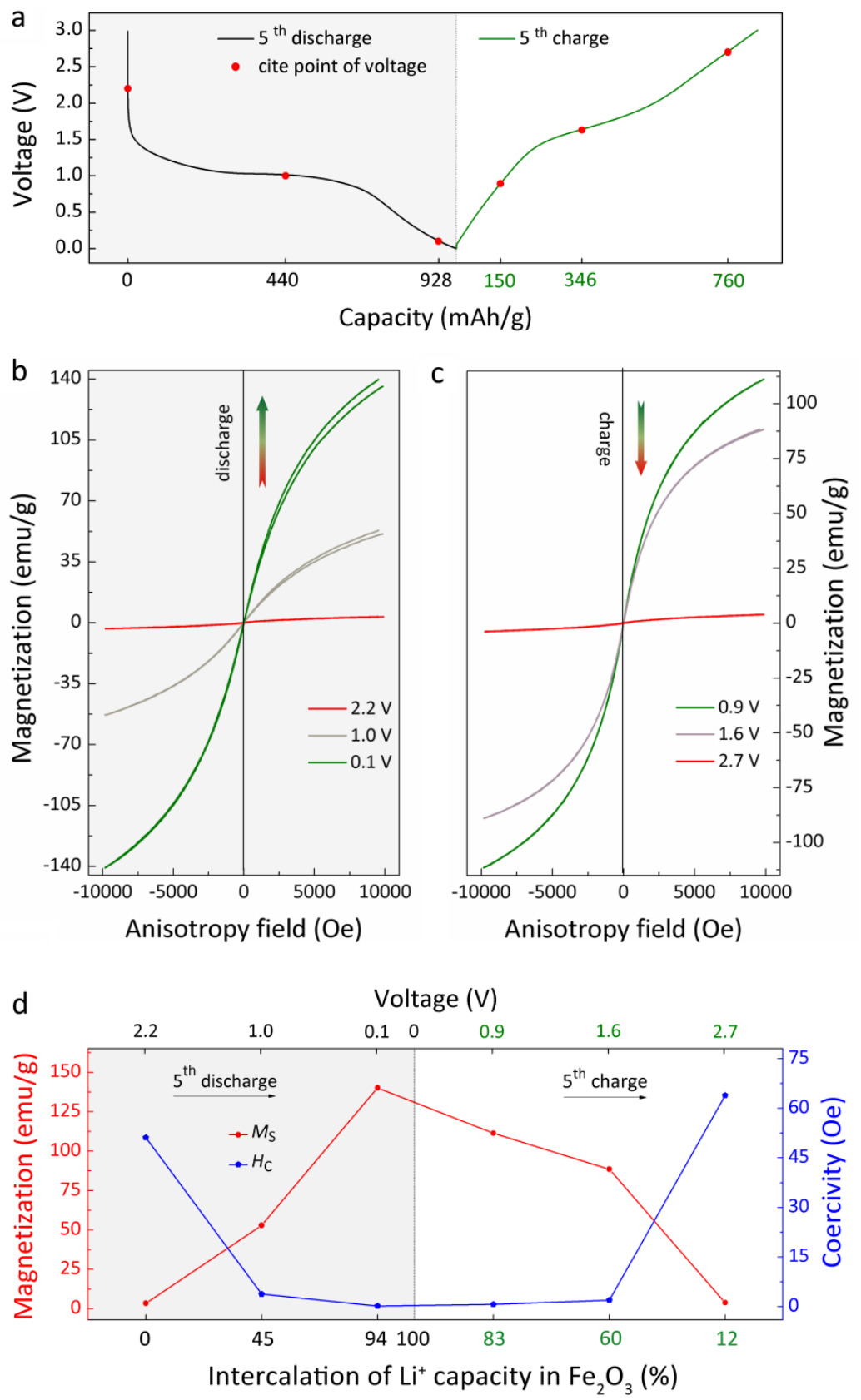

Figure S6. (a) The fifth discharge-charge curve recorded at the selected voltage points for the magnetization manipulation during the fifth lithiation-delithiation process. (b,c) Magnetic hysteresis loops of the experimental device being discharged and charged at different voltages in the fifth lithiation and delithiation processes, respectively. (d) Variation trend of the magnetization saturation and coercivity of the experimental device at different periods during the fifth discharge-charge process. 
Table S1 The specific saturation magnetization and coercivity of the three pristine samples collected from the magnetic hysteresis loops in Figure S1.

\begin{tabular}{lcc}
\hline Pristine materials & $\boldsymbol{M}_{\mathbf{S}}(\mathbf{e m u} / \mathbf{g})$ & $\boldsymbol{H}_{\mathbf{C}}(\mathbf{O e})$ \\
\hline Raw $\boldsymbol{\alpha}-\mathrm{Fe}_{2} \mathbf{O}_{3}$ nanoparticles & 0.862 & 202.06 \\
Electrode paste & 0.893 & 191.55 \\
Device in $\mathbf{1}^{\text {st }}$ discharge at $\mathbf{3 . 0} \mathrm{V}$ & 0.900 & 184.85 \\
\hline
\end{tabular}

Table S2 The remainder amount change of $\mathrm{Fe}_{2} \mathrm{O}_{3}$ related to XRD spectrum during first discharge process.

\begin{tabular}{llllll}
\hline Discharge voltage $(\mathrm{V})$ & $\mathbf{3 . 0}$ & $\mathbf{1 . 0}$ & $\mathbf{0 . 7}$ & $\mathbf{0 . 6}$ & $\mathbf{0 . 1}$ \\
\hline Remainder $\mathrm{Fe}_{2} \mathrm{O}_{3}(\mathrm{mg})$ & 1.10 & 0.98 & 0.56 & 0.18 & 0.00 \\
\hline
\end{tabular}




\section{Supplementary References}

(1) Wang, Y. Xu, J. Wu, H. Xu, M. Peng, Z. Zheng, G. J. Mater. Chem 2012, 22, 21923-21917.

(2) Reddy, M. V. Yu, T. Sow, C. H. Shen, Z. X. Lim, C. T. Subba Rao, G. V. Chowdari, B. V. R. Adv. Funct. Mater. 2007, 17, 2792-2799.

(3) Ma, J. Lian, J. Duan, X. Liu, X. Zheng, W. ACS J. Phys. Chem. C. 2010, 114, 10671-10676.

(4) Wu, C. Yin, P. Zhu, X. OuYang, C. Xie, Y. J. Phys. Chem. B. 2006, 110, 17806-17812.

(5) Chen, J. Xu, L. Li, W. Gou, X. Adv. Mater. 2005, 17, 582-586.

(6) Brandt, A. Winter, F. Klamor, S. Berkemeier, F. Rana, J. Pöttgen, R. Balducci, A. J. Mater. Chem. A. 2013, 1, 11229-11236. 